\title{
STUDY OF ECG AND ECHOCARDIOGRAPHIC FINDINGS IN COPD PATIENTS IN A TERTIARY CARE CENTRE
}

\author{
Ramakrishna Rachakonda1, Suryakumari Beri², Kalyankumar P. V3 \\ 1 Professor and HOD, Department of Pulmonology, Katuri Medical College. \\ 2Post Graduate, Department of Pulmonology, Katuri Medical College, Guntur. \\ ${ }^{3}$ Associate Professor, Department of Pulmonology, Katuri Medical College, Guntur.
}

\begin{abstract}
COPD is a major cause of death worldwide. Early identification of the complications, particularly pulmonary hypertension and right ventricular dysfunction can prevent or delay long-term complications.
\end{abstract}

\begin{abstract}
AIM OF THE STUDY
To study ECG and Echocardiographic profile among COPD patients and study their importance in the management of COPD.
\end{abstract}

\section{MATERIALS AND METHODS}

We have studied $103(\mathrm{~N}=103)$ patients diagnosed as COPD in our tertiary care centre. We categorized them into mild (FEV1/FVC $<0.7$ and FEV1 is $>80 \%$ predicted), Moderate (FEV1/FVC $<0.7$ and FEV1 is between $50-80 \%$ predicted). Severe (FEV1/FVC $<0.7$ and FEV1 between $30-50 \%$ of predicted) and Very Severe (FEV1/FVC $<0.7$ and FEV1 is $<30 \%$ predicted). Mild group included 6 patients, moderate group 90 patients. Severe group consisted of 5 patients and very severe group consisted of 2 patients. We studied the ECG and Echocardiographic profile of the moderate, severe and very severe group patients totalling 97.

\section{RESULTS AND CONCLUSIONS}

P pulmonale was present in $19.58 \%$. R/S ratio < 1 in V6 was seen in $15.55 \%$ of the patients. R/S ratio $>1$ was seen in $4.44 \%$. Atrial ectopics were seen in $18.55 \%$ and ventricular ectopics were seen in 3.09\%. Right axis deviation was seen in 5.55\% and no ECG changes in 39.17\%. Echocardiogram showed consistent abnormal findings in 94\%. Pulmonary arterial hypertension was seen in 94.84\%, RV hypertrophy was seen in 26.82\%. Cardiac arrhythmias and cardiac failure were seen in $8.24 \%$ each. ECG and Echocardiography can identify early pulmonary hypertension and right ventricular dysfunction. Proper institution of therapy can prevent long-term complications of severe pulmonary hypertension and right heart failure and can prolong the life and improve quality of life among COPD patients.

\section{KEYWORDS}

COPD, Electrocardiography, Echocardiography, Right Ventricular Dysfunction, Cor pulmonale, P Pulmonale, Atrial Ectopics, Right Axis Deviation, RV Hypertrophy.

HOW TO CITE THIS ARTICLE: Rachakonda R, Beri S, Kalyankumar PV. Study of ECG and echocardiographic findings in COPD patients in a tertiary care centre. J. Evolution Med. Dent. Sci. 2016;5(24):1276-1280, DOI: 10.14260/jemds/2016/299

\section{INTRODUCTION}

Chronic Obstructive Pulmonary Disease (COPD) is a major cause of chronic morbidity and mortality throughout the world. COPD is 4 th leading cause of death in the world and becomes 3 rd leading cause of death by 2030 worldwide. COPD accounts for a substantial number of visits to general physician, emergency department, hospital admissions and also a cause for frequent absence from work. COPD is associated with considerable morbidity and a patient of COPD causes financial burden to the family as well as to the national health expenditure. It is necessary to diagnose the disease early and identify patients who are likely to develop complications of pulmonary hypertension, right ventricular hypertrophy and cor pulmonale to prevent long-term complications, promote longevity and improve quality of life.

Financial or Other, Competing Interest: None.

Submission 07-02-2016, Peer Review 06-03-2016,

Acceptance 11-03-2016, Published 22-03-2016.

Corresponding Author:

Dr. Ramakrishna Rachakonda,

Sanjeevani Hospital, A1,

Ramkuteer Majestic, 3/13 Brodipet,

Guntur.

E-mail: ramakrishna45@yahoo.co.in

DOI: $10.14260 /$ jemds/2016/299
In this study, we made an attempt to see the importance of ECG and echocardiography in the evaluation of COPD patients and early recognition of complications. LV ejection fraction of $<50 \%$ were seen in $36.08 \%$. Echo identified more percent of patients with RV dysfunction compared to ECG. We propose that it is necessary to treat a COPD both by Cardiologist and Pulmonary Physician and all modalities of examination clinical, radiological, spirometry, ECG and echocardiogram together can help in identifying pulmonary hypertension to prevent long-term complications.

\section{ABBREVIATIONS}

COPD: Chronic Obstructive Pulmonary Disease; ECG: Electrocardiography; FEV1: Forced Expiratory Volume 1; FVC: Forced Vital Capacity; LVDF: Left Ventricular Dysfunction; TAPSE: Trans-tricuspid Annular Plane Systolic Excursions; PASP: Pulmonary Arterial Systolic Pressure; TTPG: Transtricuspid pressure gradient; EF: Ejection Fraction; RAE: Right Atrial enlargement; RVD: Right Ventricular Dysfunction; RHC: Right Heart Catheterization.

\section{AIMS AND OBJECTIVES}

1. To study the electrocardiographic and echocardiographic findings in COPD. 
2. To correlate these findings in assessing the severity of the disease.

3. To analyse these findings in assessing right ventricular dysfunction.

\section{Type of Study}

Prospective observational study from 2014 August to 2015 August.

\section{Inclusion Criteria}

Adult males and females aged more than 35 years with a history suggestive of chronic obstructive pulmonary airway disease were selected at random from the Outpatient Department of KMCH, Guntur, for the present study.

The diagnosis of chronic obstructive pulmonary disease is made by symptoms in the history and confirmed by physical examination, radiographic examination and lung spirometry for airway obstruction by Spiroanalyzer.

\section{Exclusion Criteria}

Cases excluded from the present study are those with a primary diagnosis of bronchial asthma, known sleep apnoea, lung cancer, known left ventricular dysfunction, other debilitating cancers, poorly controlled hypertension, significant valvular disease and known coronary artery diseases (Angina, Ischaemic changes in resting ECG or documented history of myocardial infarction).

Very poor echogenic subjects in whom meaningful echocardiographic examination could not be performed were also excluded from the present study. Cases with nonvisualisation or poor visualisation of only one or few parameter being studied in the presence of recordable readings of the other parameters are included. Patients with active pulmonary Koch's or history of old pulmonary Koch's were excluded from the study.

\section{METHODOLOGY}

After recruitment for the study, a thorough physical examination is done and routine investigations were carried out. The patients were subjected to the following examination. The patients who are diagnosed as having chronic obstructive pulmonary disease as per GOLD guidelines with FEV1/FVC $<70 \%$ or less are further divided into 3 groups.

Group I: Patients having FEV/FVC 0.7 or less but having a FEV1 of more than 80; Group II: Patients selected fulfil the above criteria and belong to moderate COPD based on predicted FEV1 (50-80\% of predicted value); Group III: Patients selected fulfil the above criteria and belong to severe COPD based on predicted FEV1 (30-50\% of predicted value); Group IV: Patients selected fulfil the above criteria and belong to very severe COPD based on predicted FEV1 $(<30 \%$ of predicted value).

\section{Radiographic Examination}

Chest X-ray postero-anterior view and lateral view obtained to detect right heart enlargement and/or pulmonary artery dilatation.

\section{Electrocardiographic Assessment}

A standard 12-lead electrocardiography obtained for each using a portable ECG machine. The following criteria are used to detect right ventricular involvement:

a. P-pulmonale pattern (P wave $>2.5 \mathrm{~mm}$ ) in leads II, III, avF; b. Right axis deviation of QRS complex; c. R/S amplitude ratio in V6 is less than $1 ; \mathrm{d}$. $\mathrm{R} / \mathrm{S}$ amplitude ratio in $\mathrm{V} 1$ more than 1; e. Clockwise rotation of the electrical axis; f. Right bundle branch block; g. S1, Q3 or S1, S2, S3 patterns.

Thorough echocardiography was performed with the help of Department of Cardiology.

This paper deals with ECG and Echocardiographic findings among patients of COPD belonging to different categories. Clinical, spirometric and radiological parameters were correlated in a different paper.

Among the 103 patients examined, 6 patients belonged to mild COPD with an FEV1 of $>80 \%$ predicted; 90 patients belonged to moderate category with FEV1 values after bronchodilator therapy between $50-80 \%$ of predicted value, 5 patients belonged to severe category with post bronchodilator FEV1 between 30 and $50 \%$ of predicted value and 2 patients belonged to very severe category with post bronchodilator FEV1 less than $30 \%$ of predicted value.

\section{Electrocardiographic Findings}

In the present study, out of total 103 patients 6 patients belong to mild category and we studied 97 patients belonging to moderate, severe and very severe groups. Among them 59/97 $(60.82 \%)$ patients showed ECG changes. The most common abnormality observed was ' $\mathrm{P}$ ' pulmonale seen in 19/97 patients $(19.58 \%)$. ' $\mathrm{P}$ ' pulmonale was present in moderate group in $17.77 \%$. In severe COPD, one of five subjects had $\mathrm{P}$ pulmonale $(20 \%)$. In very severe group, both the patients had $\mathrm{P}$ pulmonale $(100 \%)$. Prevalence of $\mathrm{P}$ pulmonale increased with severity of COPD, but in our study only 7 patients belonged to severe and very severe group. The R/S ratio in V6 $<1$ correlated significantly with echo findings of RV dysfunction; $14 / 90$ (15.55\%) in moderate COPD group had this abnormality, but none in severe and very severe groups. In our study, Atrial (18.55\%) and Ventricular ectopics (3.09\%) were seen more in the moderate group of COPD patients.

\section{Analysis of Echocardiographic Findings}

In the present study, $22.68 \%(22 / 97)$ of the patients had echocardiographic evidence comprising of RV dilatation, RV hypertrophy, RA dilatation or interventricular septum motion abnormality; $17 / 90$ (18.88\%) in the moderate group $3 / 5$ $(60 \%)$ in severe group and $2 / 2(100 \%)$ in very severe group. In the present study, majority of the patients belong to moderate group and percentage of right ventricular changes increased with severity of COPD, but as the number in the severe and very severe group is small the values are not statistically significant.

\section{Incidence of Pulmonary Hypertension in Echo- cardiographic Examination}

In the moderate group, in our study $77 / 90$ patients showed pulmonary hypertension by Echo. Out of 97 symptomatic patients, 82 have PAH and most of them had moderate COPD. In this group, mild PAH was seen 42/90 (46.66\%), moderate $\mathrm{PAH}$ was seen in $21 / 90$ (23.33\%) and severe PAH was seen in $14 / 90(15.55 \%)$. In the severe COPD group, $2 / 5$ (40\%) had mild PAH and $1 / 5$ had severe PAH (20\%). In the very severe group of 2 patients, both the patients were recorded to have moderate pAH (100\%).

The relatively low incidence of pulmonary hypertension in the severe and very severe group in the present study is because of relatively small study group belonging to severe and very severe COPD. But the incidence in the moderate and the severe groups taken together is found to be fairly 
correlating. Echocardiographic findings in our study included mild-to-severe pulmonary hypertension, RV hypertrophy (26.82\%), cardiac arrhythmias (8.24\%) and cardiac failure (8.24\%).

\section{Comparison of ECHO with ECG}

In the study group of 103 patients and 97 belonging to moderate, severe and very severe categories, ECG changes were seen only in 59 (60.82\%), p pulmonale seen in 19 (19.58\%); but Echo revealed mild PAH in 44/97 (45.36\%), moderate PAH in 23/97 (23.71\%), severe PAH in 15 (15.46\%). ECG showed R/S in V6<1 in 14/97 (14.43\%), Echo showed right ventricular hypertrophy in 22 (22.66\%), other parameters are equally comparable in both ECG and Echo. Our study showed that Echo is more sensitive in analysis of $\mathrm{PAH}$ and RVH. Other parameters like atrial and ventricular ectopics, ischemic changes were comparable in both ECG and Echo.

\section{DISCUSSION}

Presence of $\mathrm{P}$ pulmonale in ECG, R/S ratio less than was significantly correlated with RV dysfunction by echo, but less so with R/S ratio $>1$ in V1. Several studies showed the importance of P pulmonale in ECG and correlated with severity of COPD. Biljana Lazović Study (14.5\%). ${ }^{1}$ Hina Banker Study (35\%). ${ }^{2}$ Jayadev S Mod et al. Study (45\%). ${ }^{3}$ Sandeep Krishna Nalabothu Study (20\%). ${ }^{4}$ Vineeth Alexander Study (52.5\%). ${ }^{5}$ showed variable percentage of $\mathrm{P}$ pulmonale in different studies. This variation is because of variation in severity of COPD. Our study involved relatively stable people and a majority of them belonged to moderate group.

In other studies, Saratkumar Reddy et al. ${ }^{6}$ found other parameters like atrial ectopics, ventricular ectopics, incomplete RBBB, complete RBBB and atrial fibrillation and arrhythmias were seen in more than $50 \%$ of their patients. Less common findings include ST segment depression in lead II, III, aVF, T wave inversion in leads V1-V3, Right Bundle Branch Block (RBBB), S wave in lead I sign, ventricular ectopics, multifocal atrial tachycardia and S-I, S-II, S-III pattern $^{4}$ in moderate COPD. Ramabhishek Sharma Study. ${ }^{7}$ showed P pulmonale is indicator of severe COPD. ${ }^{7}$ with few false positives. Combination of rS pattern in lead V5-V6, right axis deviation, clockwise rotation, dominant $\mathrm{R}$ in lead aVR and "P" pulmonale were indicative of RVH in patients lacking classical RVH changes in ECG. ${ }^{7}$ Humagain $\mathrm{S}$ et al. Study. ${ }^{8}$ showed that amplitude of $\mathrm{p}$ wave is increased in II, III and avf leads in high PASP (>30 mm Hg) in moderate COPD patients.

\section{Normal ECG}

Normal ECG was found in 35 out of 97 patients (39.17\%). Similar observation was seen in the studies of Biljana Lazovic Study. ${ }^{1}$ Ramabhishek Sharma Study ${ }^{7}$ showed ECG changes correlated with severity of COPD. But these ECG changes are not universal. Similar views were echoed by D. Radhakrishnan et al. ${ }^{9}$ Study. Miriam J. Warnier et al. ${ }^{10}$ Study showed that abnormal ECGs were more prevalent in COPD patients (50\%) than in patients without COPD $(36 \%, \mathrm{p}=0.054)$.

Dr. Deepak Gupta Study. 11 found that the most common ECG changes were $\mathrm{P}$ wave axis $>+90^{\circ}, \mathrm{QRS}$ axis $>+90^{\circ}, \mathrm{P}$ wave height $>2.5 \mathrm{~mm}$ in lead II and $\mathrm{R}$ wave in $\mathrm{V} 6<5 \mathrm{~mm}$. There was significant negative correlation between FEV1, FEV1/FVC ratio and ECG changes; $4 \%$ of patients showed RBBB and $2 \%$ of patients showed $7 \mathrm{~mm} \mathrm{R}$ wave in V1. The observation is similar to our study and we found more patients of COPD with
$\mathrm{R} / \mathrm{S}<1$ in V6 (14.43\%) than patients with $\mathrm{R} / \mathrm{S}$ ratio $>1$ in $\mathrm{V} 1$ (6.66\%). Similar views were echoed in Sandeep Nallabothu Study. ${ }^{4}$ and Vineet Alexander Study. ${ }^{5}$

Satish Kinagi Study.12 found $18 \%$ patients had concomitant coronary artery disease; $12 / 72$ had $2+3+$ avf leads with $\mathrm{P}$ wave amplitude $>9 \mathrm{~mm}$ and he stated that this is one of the indications for long-term oxygen therapy as per the American Thoracic Society.

Vertical heart position was observed in $65 \%$ of patients of Hina Banker Study. $25 \%$ of patients in this study showed dominant S wave in V5 and V6 and in our study only 14.4 percent showed this sign in ECG. Jayadev S. Mod et al. Study. ${ }^{3}$ revealed that electrocardiographic changes in chronic cor pulmonale are due to vertical position of the heart or right ventricular hypertrophy or both.

Satish Kinagi Study. ${ }^{12}$ showed that the complications of COPD like pulmonary hypertension, cor pulmonale were better found by ECG and echocardiogram. Lokendra Dave study. ${ }^{13}$ revealed that the stage of COPD is proportional to prevalence and severity of Pulmonary Artery Hypertension (PAH). Electrocardiogram (ECG) can be used for screening of COPD with most common parameters observed may be right axis deviation, $\mathrm{P}$ pulmonale and right ventricular hypertrophy. ECG and Echo being non-invasive are more useful than invasive procedures like right heart catheterization. Vikram B Vikhe.14 Study showed that echocardiography is more sensitive than electrocardiography in detecting PAH and right ventricular dysfunction in COPD. Our study also showed echocardiography is more sensitive than ECG in identifying early Pulmonary Hypertension and Right Ventricular Dysfunction. Non-invasive and easily available can be routinely recommended in COPD.

\section{Role of Echocardiography in Assessing the severity of COPD}

Jayadev S. Mod Study. ${ }^{3}$ revealed that in comparison with ECG, 2D Echo Echocardiography can detect RV measurements, interventricular septum thickness, but cannot measure accurately RV ejection fractions. 2D Echo is also many a times inaccurate to detect pulmonary hypertension in COPD patients. NK Gupta Study. ${ }^{15}$ showed $50 \%$ of patients had normal echocardiography. (Measurable tricuspid regurgitation), pulmonary hypertension with measurable SPAP of $>30 \mathrm{mmHg}$ were found in majority in this study. Similar views were explained in the studies of Asif Hasan. ${ }^{16}$ and Shresta. ${ }^{17}$ Chetan Rathi. ${ }^{18}$ stressed the importance of echocardiography in mild-to-moderate COPD patients to detect early changes of pulmonary arterial hypertension and explained that study of PASP and TAPSE (Trans-tricuspid annular plane systolic Excursions). Were the most sensitive indicators of RV function and pulmonary hypertension.

Apart from the changes of pulmonary hypertension Agarwal KC. ${ }^{19}$ showed LVDF in a significant number of patients and according to him echocardiographic changes of pulmonary arterial hypertension and RV dysfunction correlated with severity of COPD by MMRC scale. Medhat Soliman et al. ${ }^{20}$ found that sensitivity of echo was high, but less specific and $74 \%$ of the patients had $>10 \mathrm{mmHg}$ difference between PASP calculated by echo and measured by RHC.

Radhakrishnan et al. ${ }^{9}$ showed Echocardiographic examination is reliable in following COPD patients with PAH instead of repeated cardiac catheterization. MA Higham Study. ${ }^{21}$ stressed the importance of Doppler Echocardiography 
in COPD patients and measurement of trans-tricuspid pressure gradient and that there was significant correlation with trans-tricuspid pulmonary gradient and FEV1 and DLCO. Other studies that correlated the importance of Echocardiography in COPD patients include Lokendra Dave Study. ${ }^{13}$ Vikram B Vikhe Study. ${ }^{14}$ Vineet Alexander Study. ${ }^{5}$ Bhupendrakumar Study. 22 and Jain et al. Study. 23

Our study showed lesser incidence of $\mathrm{P}$ pulmonale, because majority of our patients presented in moderate COPD stage. P pulmonale has been used as an indirect evidence of right ventricular hypertrophy by various authors.,8 Others regarded it as a positional change due to hyperinflation, lowering of diaphragm and vertical position of the heart. ${ }^{3} \mathrm{R} / \mathrm{S}$ ratio in $V^{6}<1$ : was seen in a significant number of moderate COPD patients. Right axis deviation was seen in 4 patients belonging to moderate group (4.96\%). Other changes like atrial and ventricular ectopics were found to be nonspecific and could not strictly assess the severity of COPD.

The reason for the differences in the ECG findings in our study may be due to the fact that the sample size was small and also as we had categorized the patients to different groups compared to the other studies, which were carried out on large number of patients without categorization and further our study correlated with other studies in right ventricular hypertrophy, right axis deviation, right bundle branch block and $P$ pulmonale when we correlated these findings in severe and very severe COPD patients, but most of our patients belonged to moderate COPD. Other manifestations like RBBB (Prolonged QRS $>0.10 \mathrm{sec}$, broad terminal $\mathrm{S}$ wave in lead 1 , V5, V6 and RSR complex in V1 are also seen in about 10 of 97 $(10.3 \%))$.

Correlation of ECG Findings with Severity of the Disease In the present study, the incidence of all the ECG findings increased as the severity of the disease increased. Increased incidence of 'p' pulmonale, right axis deviation and RVH were observed in patients with moderate COPD because majority of our patients belonged to moderate COPD group.

The classical view of the development of heart failure in patients with COPD is that hypoxia leads to pulmonary hypertension, which imposes increased work on the right ventricle, leading to right ventricular hypertrophy and eventually RV dilatation and then RV failure. The RA dilatation is a consequence of systolic overload on RA due to RV hypertrophy.

In our study of 97 patients belonging to moderate, severe and very severe categories Echo showed $\mathrm{EF}<50 \%$ in 35/97 $(36.08 \%)$ patients. A number of studies stressed the importance of looking for LV systolic and diastolic dysfunction in Echo as LV dysfunction in COPD patients is an important comorbidity, which determines the outcome of the disease. 12 Additional parameters studied in other studies include PASP, PADP, TAPSE, measurements which can possibly detect right ventricular dysfunction much earlier.

ECG and Echo may be normal in a number of subjects of COPD. Thorough history taking, clinical examination, spirometry, chest X-ray, arterial blood gases, 6-minute walk testing, ECG, Echocardiography together can assess a patient of COPD in identifying early pulmonary hypertension and right ventricular dysfunction leading to increased symptomatology and complications altering prognosis. Among our patients studied, 38/97 (39\%) showed normal ECG, but only less than $10 \%$ of the symptomatic group showed normal Echo indicating that Echocardiography is a more sensitive indicator. Examination of high risk patients with above investigations can probably identify early pulmonary HTN. We can help such patients in stopping smoking and instituting proper therapy, so that long-term complications are prevented and longevity improved.

\begin{tabular}{|c|c|c|c|c|c|}
\hline $\begin{array}{c}\text { ECG } \\
\text { Findings }\end{array}$ & $\begin{array}{c}\text { Moderate } \\
\text { COPD (n=90) }\end{array}$ & $\begin{array}{c}\text { Severe } \\
\text { COPD (n=5) }\end{array}$ & $\begin{array}{c}\text { Very Severe } \\
\text { COPD (n=2) }\end{array}$ & Total & P value \\
\hline P pulmonale & $16 / 90(17.77 \%)$ & $1(20 \%)$ & $2(100 \%)$ & $19(19.58 \%)$ & $<0.01$ \\
\hline Atrial ectopics & $17 / 90(18.88 \%)$ & $1(20 \%)$ & 0 & $18 / 97(18.55 \%)$ & $>0.05$ \\
\hline Ventricular ectopics & $3 / 90(3.33 \%)$ & 0 & 0 & $3(3.09 \%)$ & $>0.05$ \\
\hline R/S in V6<1 & $14 / 90(15.55 \%)$ & 0 & 0 & $14 / 97(14.43 \%)$ & \\
\hline R/S in V1>1 & $4 / 90(4.44 \%)$ & $2(2.22 \%)$ & 0 & $6(6.66 \%)$ & \\
\hline Right axis deviation & $5 / 90(5.55 \%)$ & 0 & 0 & $5 / 97(5.15 \%)$ & \\
\hline No ECG changes & $35 / 90$ & 3 & 0 & $38 / 97(39.17 \%)$ & \\
\hline \multicolumn{7}{|r|}{ Table 1: ECG Changes in COPD Patients } \\
\hline
\end{tabular}

\begin{tabular}{|c|c|c|c|c|c|c|}
\hline $\begin{array}{c}\text { ECHO } \\
\text { Findings }\end{array}$ & $\begin{array}{l}\text { Moderate } \\
\text { COPD }\end{array}$ & $\begin{array}{c}\text { Severe } \\
\text { COPD }\end{array}$ & $\begin{array}{c}\text { Very Severe } \\
\text { COPD }\end{array}$ & Total & $\begin{array}{c}\begin{array}{c}\text { Percentage } \\
\mathrm{N}=97\end{array} \\
\end{array}$ & P Value \\
\hline Mild PAH & $42 / 90(46.66 \%)$ & $2 / 5(40 \%)$ & $0 / 2$ & $44 / 97(45.36 \%)$ & $45.36 \%$ & $<0.01$ \\
\hline Moderate PAH & $21 / 90(23.33 \%)$ & 0 & $2 / 2$ & $23 / 97(23.71 \%)$ & $23.71 \%$ & $<0.01$ \\
\hline Severe PAH & $14 / 90(15.55 \%)$ & $1 / 5(20 \%)$ & $0 / 2$ & $15 / 97(15.46 \%)$ & $15.46 \%$ & $<0.01$ \\
\hline PAH $\%$ & $77 / 90(85.85 \%)$ & $3 / 5(60 \%)$ & $2 / 2(100 \%)$ & $92 / 97(94.84 \%)$ & $94.84 \%$ & \\
\hline RV Hypertrophy & $17 / 90(18.88 \%)$ & $3 / 5(60 \%)$ & $2 / 2(100 \%)$ & $22 / 97(26.82 \%)$ & $26.82 \%$ & $<0.002$ \\
\hline Cardiac Arrhythmias & $7 / 90(7.77 \%)$ & $1 / 5(20 \%)$ & 0 & $8 / 97$ & $8.24 \%$ & $<0.05$ \\
\hline Cardiac Failure & $7 / 90(7.77 \%)$ & $1 / 5(20 \%)$ & 0 & $8 / 97$ & $8.24 \%$ & \\
\hline
\end{tabular}

Correlation of Echocardiographic findings with severity of the disease.

\section{SUMMARY AND CONCLUSIONS}

COPD is a disease of old age 6th and 7th decades and most of patients have FEV1 between 50\%-80\%. ECG changes significantly correlated with low values of FEV1/FVC ratio.
Echocardiography is a very useful noninvasive investigation to assess pulmonary hypertension and right ventricular hypertrophy. It is very sensitive even in mild-to-moderate COPD patients. Echo is more sensitive compared to ECG. It is 
important to assess a patient of COPD not only with clinical, radiological, spirometric criteria, but also with ECG and Echo findings as later parameters can assess the severity and outcome of the disease better. Based on all these available parameters a more aggressive approach to treat the COPD patients can be taken, so that the onset of cor pulmonale would be delayed as long as possible.

All the modalities of investigation should be considered together, as there is a possibility of false negatives in individual investigations. Our study and several other studies showed. ${ }^{1}$ ECG and Echocardiography can be normal in a significant number of patients of COPD. Correlation of pulmonology and cardiology teams is necessary in diagnosing and treating these patients as good number of COPD patients have associated coronary artery disease and LVDF. ${ }^{18}$ which can be devastating individually as well as a comorbidity along with COPD. Echocardiography is a suitable noninvasive alternative to right heart catheterization.

\section{REFERENCES}

1. Lazović B, Svenda MZ, Mazić S, et al. Analysis of electrocardiogram in chronic obstructive pulmonary disease patients. Med Pregl 2013;66(3-4):126-129.

2. Hina Banker, Anita Verma. Electrocardiographic changes in COPD. NHL Journal of Medical Sciences 2013;2(2):5558.

3. Jayadev S Mod, Parthavi Khandhar, Kanhai Lalani. Ecg changes in chronic cor pulmonale. Indian Journal of Applied Research 2014;4(12):ISSN-2249-555X.

4. Sandeep Krishna Nalabothu, Leela Krishna Kaku. A study of electrocardiographic changes in chronic obstructive pulmonary disease. Sch J App Med Sci 2015;3(1G):470472. ISSN 2320-6691 (Online), ISSN 2347-954X (Print).

5. Alexander V, Pajanivel R, Surendra Menon K, et al. Prevalence cardiac comorbidities and its relation to severity staging of chronic obstructive pulmonary disease. IJCRR 2015;7(17):27-33.

6. Sarath Kumar Reddy B, Lokendranath G, Prabhakar Rao R. Electrocardiographic changes in chronic obstructive pulmonary disease. Journal of Evidence Based Medicine and Healthcare 2014;1(3):111-117.

7. Ram Abhishek Sharma, Zia Hashim, Ekta Sharma, et al. Teerthanker mahaveer medical college \& research center, moradabad, diagnosis of severity of COPD on the basis of electrocardiogram. Indian Journal of Basic \& Applied Medical Research 2013;6(2):527-530.

8. Humagain S, Keshari S, Gurung R, et al. Electrocardiographic changes in chronic obstructive pulmonary disease patients with elevated pulmonary artery systolic pressure. Nepalese Heart Journal 2011;8(1):12-15.

9. Radha Krishnan D, Barama Srihari. A study on the severity of right ventricular dysfunction in correlation with the severity of lung dysfunction in chronic obstructive pulmonary disease patients-COPD. The American Journal of science and medical research 2015;1(1):112-119. ISSN: 2377-6196.

10. Miriam J Warnier, Frans H Rutten, Mattijs E Numans, et al. Electrocardiographic characteristics of patients with chronic obstructive pulmonary disease. Journal of Chronic obstructive Pulmonary disease, COPD 2013;10:62-71. ISSN: 1541-2555 print / 1541-2563.
11. Deepak Gupta, Pradeep Agrawal, Kothari RP, et al. Electrocardiographic changes in chronic obstructive pulmonary disease-correlation with air flow limitation. IOSR Journal of Dental and Medical Sciences (IOSR-JDMS) 2015;14(9):49-52. e-ISSN:2279-0853, p-ISSN:2279-0861. www.iosrjournals.org.

12. Sathish kinagi, Sharan Patil, Sayeeda Afiya, et al. Analysis of chronic obstructive pulmonary disease with clinical parameters, ECG, and Echo. Journal of Evolution of Medical and Dental Sciences 2014;3(57):12864-12880. DOI:10.14260/jemds/2014/3710.

13. Dave L, Rajoriya V, Dubey TN, et al. Evaluation of BODE index as a predictor of pulmonary hypertension in COPD patients. Ann Int Med Den Res 2015;1(3):213-17.

14. Vikram B Vikhe, Prakash S Shende, Rahul S Patil, et al. Cardiovascular complications in chronic obstructive pulmonary disease with reference to $2 \mathrm{~d}$ echocardiography findings. National journal of medical research 2013;3(4):385-388. print ISSN:2249-4995.| eISSN:22778810.

15. Gupta NK, Ritesh Kumar Agrawal, Srivastav AB, et al. Echocardiographic evaluation of chronic obsatructive pulmonary disease patients and its co-relation with severity of disease. Lung India 2011;28(2):105-109.

16. Asif Hasan, Uwais Ashraf M, Shirin Naaz, et al. Study of clinical and echocardiographic profile of patients of chronic obstructive pulmonary disease helping in early diagnosis of corpulmonale. International Journal of Enhanced Research in Medicines and Dental care, ISSN: 2349-1590, 2014;1(2):5-8. Available online at: www.erpublications.com.

17. Shrestha B, Dhungel S, Chokhani R. Echocardiography based cardiac evaluation in the patients suffering from chronic obstructive pulmonary disease. Nepal Med Coll J 2009;11(1):14-18.

18. Chetan Rathi, Anil Wanjari, Sourya Acharya. Pulmonary hypertension in mild-moderate COPD: an early link. Journal of Evidence based Medicine and Healthcare 2015;2(42):7330-7340.

19. Agarwal KC, Deepak Singh, Patil Shital, et al. Cardiovascular alterations in COPD what hurts the patient the most? Journal of Medical Education \& Research 2013;3(1):21-30. ISSN: 2249-9660.

20. Medhat Soliman A, Hussen Heshmat B, Yousif Amen A, et al. Detection of right sided heart changes and pulmonary hypertension in COPD patients. Egyptian Journal of Chest Diseases and Tuberculosis 2015;64(2):335-341.

21. Higham MA, Dawson D, Joshi J, et al. Utility of echocardiography in assessment of pulmonary hypertension secondary to COPD. Eur Respir J 2001;17(3):350-355.

22. Bhupendrakumar Jain, Nikhilesh Pasari, Ashok Bajpai, et al. Evaluation of right ventricular dysfunction andf pulmonary artery hypertension to secondary COPD severity by electrocardiogram and echocardiography. Journal of Evolution of Medical and dental sciences 2015;4(42):7275-7281.

23. Jain J, Soni P, Apte S, et al. A study of correlation between echocardiographic changes with the duration and severity of chronic obstructive pulmonary disease. Journal of Evolution of Medicine and Dental Sciences 2014;3(8):1997-2002. 